\title{
Non-Data-Aided Timing Acquisition for Asynchronous IDMA Systems
}

\author{
Zhao Wang • JianHao Hu • XingZhong Xiong • \\ Jie Song
}

(C) Springer Science+Business Media, LLC. 2012

\begin{abstract}
Sample-timing error can cause significant performance degradation for the interleave-division multiple-access systems (Wang et al. 2009). In this paper, we propose a nondata-aided timing acquisition scheme to mitigate sample-timing error due to the asynchronous transmission of random allocated user ends (UEs) on the uplink. A closed-loop timing control scheme is constructed for the asynchronous IDMA system in this paper. We use the extrinsic information generated during the iterative detection algorithm with signal noise ratio evolution to estimate the timing offset for the asynchronous uplink in the base station (BS) receiver. The BS receiver returns the timing control bits, which is generated with estimated timing offsets, to the corresponding UE. And the UE uses the timing control bits to adjust local transmission time to guarantee the sampling performance at the BS receiver. The simulation results show that the proposed acquisition scheme based on feedback loop can provide high acquisition probability and low false alarm probability. The proposed scheme can overcome the bit-error-rate performance bottleneck, which is caused by the sample-timing error in BS receiver due to the asynchronous signals in the uplink.
\end{abstract}

Keywords IDMA $\cdot$ Sample-timing error $\cdot$ SNR evolution $\cdot$ Non-data-aided · Timing acquisition

\footnotetext{
Z. Wang $\cdot$ J. Hu $\cdot$ X. Xiong $(\varangle) \cdot$ J. Song

National Key Laboratory of Science and Technology on Communications, University of Electronic Science and Technology of China, Chengdu, China e-mail: xzxiong@uestc.edu.cn

\section{Z. Wang}

e-mail: fallineva@uestc.edu.cn

J. $\mathrm{Hu}$

e-mail: jhhu@uestc.edu.cn

J. Song

e-mail: raul@uestc.edu.cn

X. Xiong

Sichuan University of Science and Engineering, Zigong, China
} 


\section{Introduction}

In the IDMA systems [1], the air signal, which is the overlapped version of different UEs transmission signals in frequency and time domain, is sampled at the digital front end of the BS receiver. Then the BS receiver performs the iterative detection algorithm to eliminate the multiple-access interference (MAI) and inter-symbol interference (ISI) with low computation complex. For the ideal scenario, in which the signals from different user are perfectly synchronized at the BS receiver, the IDMA systems can achieve excellent MAI and ISI cancellation performance at the BS. Recent researches [1-3] are carried out with perfect synchronization of the uplink. That is, the BS receiver can get the best sampling point for all of the UEs. However, we can not guarantee the synchronization of the received signals from different users due to the random geographic distribution of the UEs. Thus, the uplink signals in the practical IDMA systems become asynchronous. Ref. [4] studied the impact of the sample-timing error for the IDMA system and demonstrated that the performance of IDMA system is sensitive with the sample-timing error. Ref. [4] also showed that the sample-timing error can introduce severe MAI and ISI into the system to cause serious BER performance degradation. In the traditional timing synchronization schemes for the multiple-access (MA) systems, pilot, which can be pseudo-noise (PN) sequence, can help to recover timing error. However, the system has to sacrifice the capacity for the pilots. Thus, a non-data-aided synchronization scheme is studied to achieve high efficiency in this paper.

In this paper, we propose a novel timing acquisition scheme for the uplinks in the asynchronous IDMA system to achieve near best sampling point for the received signals from different users at BS. Differing from the conventional CDMA signals, the different interleaving diagram is the only way for us to distinguish each user in the BS receiver. Therefore, it is hard for the IDMA receiver to mitigate the timing error directly with the chip level information. But we can estimate the transmission timing error with SNR-variance evolution techniques [5-7]. Thus, it is a possible solution that the BS returns the estimated timing error to the corresponding user to calibrate its transmission timing [8]. We estimate the timing error for each user with the SNR-variance evolution during the iterative detection process for the uplink at the BS. And then the timing error is transmitted to the corresponding user through downlink to adjust local transmission time to achieve near perfect synchronization for uplink signals. The numerical results show that the proposed scheme can achieve near ideal synchronous performance for the multi-user applications. Because the pilot is not used in the proposed scheme, our scheme has higher system efficiency than that with pilot. To avoid inter-block interference (IBI), zero padding (ZP) scheme [9-11] is applied in our system. Perfect power control is also assumed in the system, which means that the received signal from each user has equal power. We also show that our timing acquisition scheme is simple, fast and accurate in simulation study.

The rest of this paper is organized as follows. We provide the system model and sampletiming error analysis in Sect. 2. The timing acquisition scheme is proposed in Sect. 3. We perform the simulation study for the proposed scheme in Sect. 4. Finally, we draw our conclusion.

\section{System Model and Sample-Timing Error Analysis}

\subsection{System Model}

The system model with $K$ simultaneous users is shown in Fig. 1. In the IDMA system, the input data sequence $\mathbf{d}_{k}$ of user $k$ is encoded based on a low-rate code $C$, generating sequence 


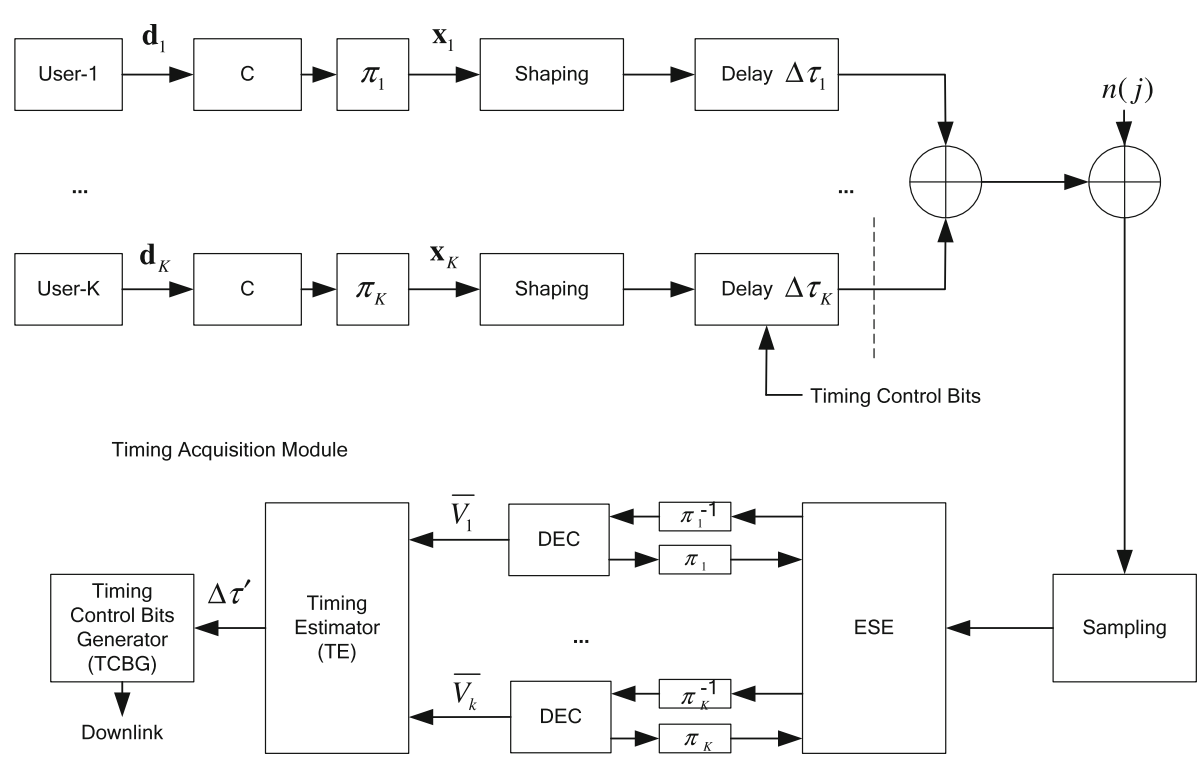

Fig. 1 Block diagram of uplink channel of asynchronous IDMA system with timing acquisition module

$\mathbf{c}_{k}=\left[c_{k}(1), \ldots, c_{k}(j), \ldots, c_{k}(J)\right]^{T}$, where $J$ is the block length. Then $\mathbf{c}_{k}$ is permutated by an interleaver $\pi_{k}$, yielding $\mathbf{x}_{k}=\left[x_{k}(1), \ldots, x_{k}(j), \ldots, x_{k}(J)\right]^{T}$. We call the elements in $\mathbf{x}_{k}$ as "chips". After base-band shaping, the signal enters the "Delay" module to simulate the asynchronous timing on the transmission. At the receiver end of the IDMA system, the received signal after sampling, $r(j)$, is used to perform iterative processing with the elementary signal estimator (ESE) and decoder (DEC) [1].

For the conventional cellular wireless system, the uplink assignment for different user is performed one after another. We apply this method in our asynchronous IDMA system. There is only one access channel for the uplink of IDMA system that can be monopolized by only one user at a certain time. That is, the multiple users can access into the IDMA system one by one. During the accessing procedure, the BS receiver estimates the timing error and returns the timing adjustment to corresponding user to achieve timing synchronization, which can be seen as a closed loop shown in Fig. 1. Thus, the accessed users all have achieved synchronization when a new user adds into the system. In our proposed system model, we use the subscript $K$ to denote the new user that tries to add into the system.

Without losing generality, we use raise cosine filter to shape the transmitted signals in the system. The shape pulse $R c(t)$ can be represented as

$$
R c(t)=\frac{\sin \left(\frac{\pi t}{T c}\right)}{\frac{\pi t}{T c}} \times \frac{\cos \left(\frac{\pi R t}{T c}\right)}{1-\frac{4 R^{2} t^{2}}{T c}}
$$

where $R$ denotes the roll-off coefficient, and $T c$ denotes the chip duration.

Now we consider that user- $K$ is trying to access into the system. In the system model, we can assume a scenario that every user in the system has perfect timing before user- $K$ is added in the system. Let $\Delta \tau_{k}$ denote the timing error of user- $K$ from the viewpoint of BS receiver. We get

$$
\Delta \tau_{k}=0, \quad \text { when } k=1,2, \ldots, K-1 .
$$


And we assume that the timing error of user- $K$ is a random variable. According to [4], we only use a fraction of $T c$ to quantify the random delay for user- $K$ in our system. Without loss of generality, we assume that $\Delta \tau_{K}$ satisfies the uniform distribution within one $T c$. That is,

$$
\Delta \tau_{K} \in U\left[\frac{-T c}{2}, \frac{T c}{2}\right]
$$

Thus, the received signal for the multiple-access system with $K-1$ simultaneous users can be presented as

$$
r(t)=\sum_{k=1}^{K-1} h_{k} x_{k}(j) R c\left(t-j T c-\Delta \tau_{k}\right)+n(t)
$$

where $x_{k}(j)$ is the $j$ th chip for user- $k$ in a block after interleaving, while $h_{k}$ represents the channel coefficient for user- $k$ and $n(t)$ is white Gaussian noise with two-sided power spectral density $N_{0} / 2=\sigma^{2}$. In order to conquer inter-block-interference (IBI), a string of zeros (ZP) is appended after information sequence $\mathbf{x}_{k}$. The length of the zero string should be larger than the summery of channel memory length and the maximum probation delay.

Now we discuss the situation that user- $K$ adds into the system, and then $r(t)$ can be represented as

$$
\begin{aligned}
r(t)= & \sum_{j=1}^{J} \sum_{k=1}^{K-1} h_{k} x_{k}(j) R c\left(t-j T c-\Delta \tau_{k}\right) \\
& +\sum_{j=1}^{J} h_{K} x_{K}(j) R c\left(t-j T c-\Delta \tau_{K}\right)+n(t)
\end{aligned}
$$

Considering (2) and (3), (5) can be rewritten as

$$
\begin{aligned}
r(t)= & \sum_{j=1}^{J} \sum_{k=1}^{K-1} h_{k} x_{k}(j) R c(t-j T c) \\
& +\sum_{j=1}^{J} h_{K} x_{K}(j) R c\left(t-j T c-\Delta \tau_{K}\right)+n(t)
\end{aligned}
$$

In the receiver, the timing estimator (TE) estimates the timing error with the mean variance of detected signal in the last block for user- $K, \overline{V_{K}}$, which can be represented by

$$
\overline{V_{K}}=\frac{1}{J} \times \sum_{j=1}^{J} \operatorname{Var}\left(x_{K}(j)\right) .
$$

The estimated timing error for the corresponding user is denoted by $\Delta \tau^{\prime}$. Then the timing control bits generator (TCBG) encodes the estimated $\Delta \tau^{\prime}$ to control bits (CB) and transmit to the UE through the downlink. 


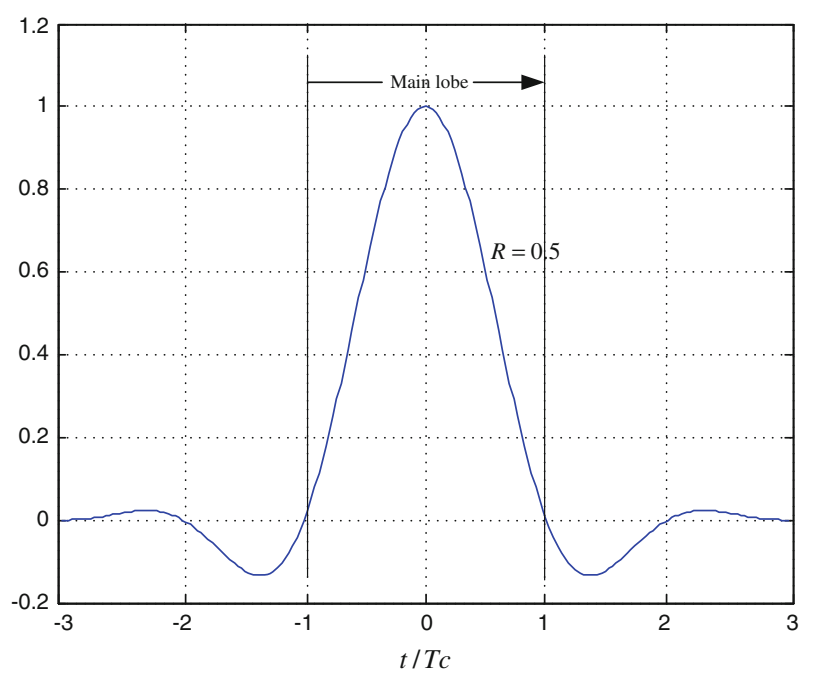

Fig. 2 The pulse shapes used in the system given by (1), where the roll-off coefficient $R=0.5$

When user- $K$ receives $\mathrm{CB}$ from the downlink, the UE adjust their transmission timing with the timing error information, $\Delta \tau^{\prime}$. After adjustment, the received signal at BS is

$$
\begin{aligned}
r(t)= & \sum_{j=1}^{J} \sum_{k=1}^{K-1} h_{k} x_{k}(j) R c(t-j T c) \\
& +\sum_{j=1}^{J} h_{K} x_{K}(j) R c\left(t-j T c-\left(\Delta \tau_{K}-\Delta \tau^{\prime}\right)+n(t)\right.
\end{aligned}
$$

The optimal adjustment of transmission timing can be represented by

$$
\Delta \tau_{K}-\Delta \tau^{\prime}=\operatorname{Dis}(K)=0
$$

where $\operatorname{Dis}(K)$ represents the synchronization performance for user- $K$ in our analysis.

In the next section, we will analyze the effect of sample- timing error in the signal detection in IDMA systems, and the idea of our synchronization scheme.

\subsection{Analysis of Sample-Timing Error}

In this part, we analyze the impact of sample-timing error on SNR-variance evolution in the iterative detection of IDMA system. Our analysis is based on the scenario that the BS receiver can achieve the ideal sample timing for all of the accessed users when user- $K$ adds into the system. That is, $\operatorname{Dis}(k)=0, k=1,2, \ldots, K-1$.

Figure 2 shows the pulse shape which we use in the system. For simplification, we can only analyze the main lobe of the pulse since the magnitude of the side lobe is very small compared with the main lobe. We use $R c_{\text {main }}(t)$ to represent the main lobe of the pulse shape. So we simplify (1) as 


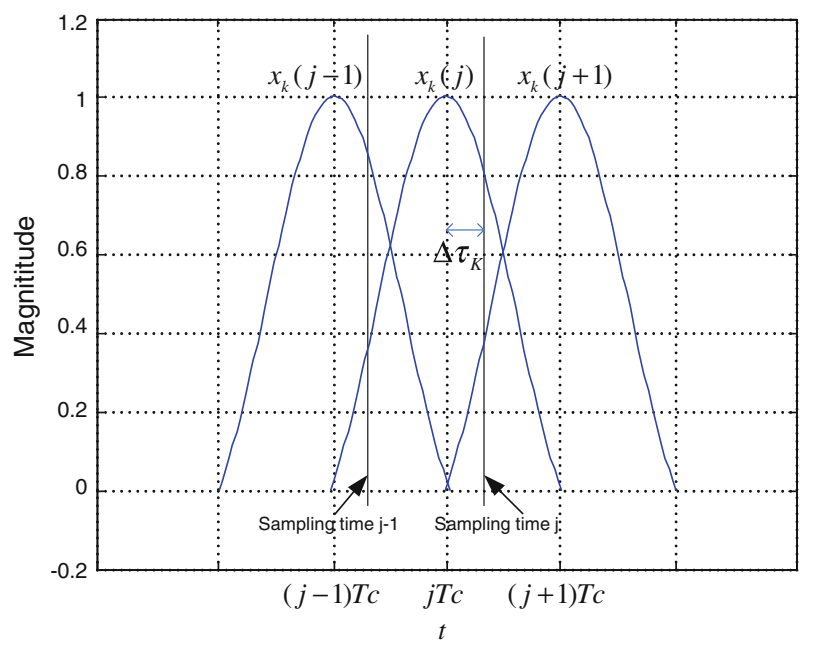

Fig. 3 The effect of timing error for sampling

$$
R c(t)= \begin{cases}R c_{\text {main }}(t)=\frac{\sin \left(\frac{\pi t}{T c}\right)}{\frac{\pi t}{T c}} \times \frac{\cos \left(\frac{\pi R t}{T c}\right)}{1-\frac{4 R^{2} t^{2}}{T c}}, t \in[-T c, T c] \\ 0, \quad \text { otherwise }\end{cases}
$$

Now we focus on the sampling procedure before ESE. According to Nyquist Theorem, we set the sampling period $T s$ equivalent to the chip duration $T c$. According to (6), we get the sampled signal as

$$
\begin{aligned}
r(j) & =\left.r(t)\right|_{t=j T c} \\
& =\sum_{k=1}^{K-1} h_{k} x_{k}(j)+\sum_{j=1}^{J} h_{K} x_{K}(j) R c\left(\Delta \tau_{K}\right)+n(j)
\end{aligned}
$$

Substituting (10) into (11), we get

$$
\begin{aligned}
r(j)= & \sum_{k=1}^{K-1} h_{k} x_{k}(j)+h_{K} R c_{\text {main }}\left(\Delta \tau_{K}\right) x_{K}(j) \\
& +h_{K} R c_{\text {main }}\left(T c-\left|\Delta \tau_{K}\right|\right) x_{K}(j \pm 1)+n(j)
\end{aligned}
$$

Equation (12) represents the sampled digital signal, which is sent into ESE. The second term on the right part of (12) represents the useful signal for user- $K$, while the other terms represent the distortion components for user- $K$. In (12), $x_{K}(j \pm 1)$ is the ISI for $x_{K}(j)$. We use the main lobe of pulse shape to approximate the whole pulse in time domain. Thus, only $x_{K}(j+1)$ or $x_{K}(j-1)$ can be the distortion components in (12), which is shown in Fig. 3.

The outputs of ESE are the extrinsic log-likelihood ratios (LLRs) of $\left\{x_{k}(j)\right\}$, which is defined below [1]:

$$
e_{\mathrm{ESE}}\left(x_{k}(j)\right) \equiv \log \left(\frac{p\left(y \mid x_{k}(j)=+1\right)}{p\left(y \mid x_{k}(j)=-1\right)}\right)
$$


With regard to (12), $\Delta \tau_{K}$ is a random variable in the practical system. But we can assume that $\Delta \tau_{K}$ is known a prior at the receiver in order to discuss the impact of timing error. So (13) can be rewritten as

$$
e_{\mathrm{ESE}}\left(x_{k}(j) \mid \Delta \tau_{K}\right) \equiv \log \left(\frac{p\left(y \mid x_{k}(j)=+1, \Delta \tau_{K}\right)}{p\left(y \mid x_{k}(j)=-1, \Delta \tau_{K}\right)}\right)
$$

Let $\zeta_{k}(j)$ denote the distortion components for user- $k$. We rewrite (12) as

$$
r(j)=\left\{\begin{array}{l}
h_{k} x_{k}(j)+\zeta_{k}(j), \quad k=1,2, \ldots, K-1 \\
h_{k} R c_{\text {main }}\left(\Delta \tau_{k}\right) x_{k}(j)+\zeta_{k}(j), \quad k=K
\end{array}\right.
$$

According to the central limit theorem, $\zeta_{k}(j)$ can be approximated as a Gaussian variable, when number of simultaneous users is large. In order to study the impact of $\Delta \tau_{K}$ for the detection algorithm, we explore the ESE detection algorithm as follows. We assume that $\Delta \tau_{K}$ is available in the detection. $E()$ and $\operatorname{Var}()$ are the mean and variance functions, respectively. Initially, we set $E\left(x_{k}(j)\right)=0$ and $\operatorname{Var}\left(x_{k}(j)\right)=1$ for $\forall k, j$, implying no extrinsic information at the beginning of the iterative detection for IDMA signals. To simplify our analysis, we only apply the single path channel in our analyzing. The similar conclusion can be obtained for the multiple path channel environments.

\section{ESE Detection Algorithm with Timing Offset in a Single Path Channel:}

Step(i): Estimation of Interference Mean and Variance

$$
\begin{aligned}
& E(r(j))=\sum_{k=1}^{K-1} h_{k} E\left(x_{k}(j)\right)+h_{K} R c_{\text {main }}\left(\Delta \tau_{K}\right) E\left(x_{K}(j)\right) \\
& +h_{K} R c_{\text {main }}\left(T c-\left|\Delta \tau_{K}\right|\right) E\left(x_{K}(j-1)\right)+n(j), \\
& \operatorname{Var}(r(j))=\sum_{k=1}^{K-1}\left|h_{k}\right|^{2} \operatorname{Var}\left(x_{k}(j)\right) \\
& +\left|h_{K}\right|^{2} R c_{\text {main }}^{2}\left(\Delta \tau_{K}\right) \operatorname{Var}\left(x_{K}(j)\right) \\
& +\left|h_{K}\right|^{2} R c_{\text {main }}^{2}\left(T c-\left|\Delta \tau_{K}\right|\right) \operatorname{Var}\left(x_{K}(j-1)\right)+n(j), \\
& E\left(\zeta_{k}(j)\right)=\left\{\begin{array}{l}
E(r(j))-h_{k} E\left(x_{k}(j)\right), \quad k=1,2, \ldots, K-1 \\
E(r(j))-h_{K} R c_{\text {main }}\left(\Delta \tau_{K}\right) E\left(x_{K}(j)\right), \quad k=K
\end{array}\right. \\
& \operatorname{Var}\left(\zeta_{K}(j)\right)=\left\{\begin{array}{l}
\operatorname{Var}(r(j))-\left|h_{k}\right|^{2} \operatorname{Var}\left(x_{k}(j)\right), \quad k=1,2, \ldots, K-1 \\
\operatorname{Var}(r(j))-\left|h_{k}\right|^{2} R c_{\text {main }}^{2}\left(\Delta \tau_{K}\right) \operatorname{Var}\left(x_{k}(j)\right), \quad k=K
\end{array}\right.
\end{aligned}
$$

Step(ii): LLR Generation

$$
e_{\mathrm{ESE}}\left(x_{k}(j)\right)=\left\{\begin{array}{l}
\frac{2 h_{k}\left(r(j)-E\left(\zeta_{k}(j)\right)\right)}{\operatorname{Var}\left(\zeta_{k}(j)\right)}, \quad k=1,2, \ldots, K-1 \\
\frac{2 h_{k} R c_{\operatorname{main}}\left(\Delta \tau_{K}\right)\left(r(j)-E\left(\zeta_{k}(j)\right)\right)}{\operatorname{Var}\left(\zeta_{k}(j)\right)}, k=K
\end{array}\right.
$$

Remark - Our numerical simulations showed that the performance of the accessed users (user-1 $\sim K-1$ ) decrease slightly due to the asynchronous user- $K$, when there is a large number of simultaneous users in the system.

In our study, we approximate $\operatorname{Var}\left(\zeta_{K}(j)\right)$ with the mean of $V_{\zeta_{K}}$, which is defined in (7). This pessimistic approximation leads greatly simplifies to our analysis with slightly performance sacrifices. The similar analysis method has been used in [12,13] for CDMA receiver and [1] for IDMA receiver. According to the ESE function, the output of ESE for user- $K$, $e_{\mathrm{ESE}}\left(x_{K}(j)\right)$, can be represented by 


$$
\begin{aligned}
e_{\mathrm{ESE}}\left(x_{K}(j)\right)= & \frac{\left(h_{K} R c_{\text {main }}\left(\Delta \tau_{K}\right) x_{K}(j)+\zeta_{K}(j)-E\left(\zeta_{K}(j)\right)\right)}{V_{\zeta_{K}}} \\
& \times 2 h_{K} R c_{\text {main }}\left(\Delta \tau_{K}\right) \quad j=1,2,3 \ldots, J
\end{aligned}
$$

where

$$
V_{\zeta_{k}}=\sum_{k^{\prime} \neq k}\left|h_{k^{\prime}}\right|^{2} \overline{V_{k^{\prime}}}+\left|h_{K}\right|^{2} R c_{\text {main }}^{2}\left(T c-\left|\Delta \tau_{K}\right|\right)+\sigma^{2}
$$

From (21) and (22), the average output SNR of ESE for user- $K$ for symbol $j, s n r_{K}$, is

$$
\begin{aligned}
s n r_{K}= & \frac{E\left(\left|h_{K} R c_{\text {main }}\left(\Delta \tau_{K}\right) x_{K}(j)\right|^{2}\right)}{E\left(\left|\zeta_{K}(j)-E\left(\zeta_{K}(j)\right)\right|^{2}\right)} \\
& \approx \frac{E\left(\left|h_{K} R c_{\text {main }}\left(\Delta \tau_{K}\right) x_{K}(j)\right|^{2}\right)}{V_{\zeta_{K}}} \\
= & \frac{h_{K}^{2} R c_{\text {main }}^{2}\left(\Delta \tau_{K}\right)}{\sum_{k=1}^{K-1}\left|h_{k}\right|^{2} \overline{V_{k}}}+\left|h_{K}\right|^{2} R c_{\text {main }}\left(T c-\left|\Delta \tau_{K}\right|\right)+\sigma^{2}
\end{aligned}
$$

In a quasi-static wireless channel model, the channel coefficient $h_{k}$ can be seen as constant. The $V_{k}$ can be represented as a function of $\Delta \tau_{K}$, according to Appendix A. Therefore, $s n r_{K}$ can be represented as a function of $\Delta \tau_{K}$ as follows:

$$
s n r_{K}=S\left(\Delta \tau_{K}\right), \Delta \tau_{K} \in\left[-\frac{T c}{2}, \frac{T c}{2}\right]
$$

In our study, we only discuss the monotonicity of $S\left(\Delta \tau_{K}\right)$ to simplify the analysis. For the best system performance, we should achieve the maximum SNR for ESE output. From Appendix A, we obtain that $S\left(\Delta \tau_{K}\right)$ is an even function. When $\Delta \tau_{K} \in\left[0, \frac{T c}{2}\right], S\left(\Delta \tau_{K}\right)$ is a monotonically decreasing function. And when $\Delta \tau_{K} \in\left[-\frac{T c}{2}, 0\right], S\left(\Delta \tau_{K}\right)$ is a monotonically increasing function. Thus, $S\left(\Delta \tau_{K}\right)$ gets its maximum value when $\Delta \tau_{K}=0$. We can use the shape ' $\cap$ ' to approximate the profile of function $S\left(\Delta \tau_{K}\right)$.

\subsection{SNR-Variance Evolution Technique}

In this section, we first briefly describe the SNR-variance evolution in a ZP-IDMA system. Details of SNR-variance evolution can be found in [5]. We just summarize the main results of [5]. Then we deduce the algorithm to estimate timing error with SNR-variance evolution.

In the ZP-IDMA, the received signal can be expressed in the matrix form as

$$
y=\sum_{k=1}^{K}\left[\begin{array}{ll}
\mathbf{H}_{k} & \tilde{\mathbf{H}}_{k}
\end{array}\right]\left[\begin{array}{l}
\mathbf{x}_{k} \\
0
\end{array}\right]+\mathbf{n}
$$

Let $\mathbf{H}_{k}^{Z P}=\left[\mathbf{H}_{k} \tilde{\mathbf{H}}_{k}\right]$ denote the channel coefficient matrix for ZP-IDMA system. We can rewrite Eq. (25) as

$$
y=\sum_{k=1}^{K} \mathbf{H}_{k}^{Z P}\left[\begin{array}{l}
\mathbf{x}_{k} \\
0
\end{array}\right]+\mathbf{n}
$$

We can freely select $\tilde{\mathbf{H}}_{k}$ to construct $\mathbf{H}_{k}^{Z P}$, which is a circulant matrix, because $\tilde{\mathbf{H}}_{k}$ corresponds the zero appended signals (27) and (28) shows the selected circulant matrix when $L=3$, where $L$ is the multi-path number. 


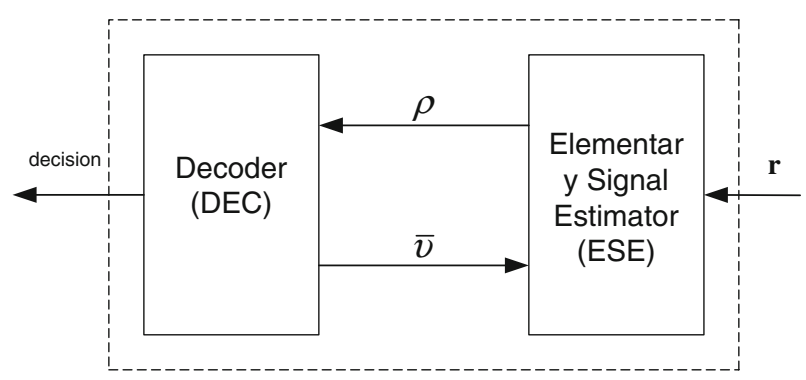

Fig. 4 The structure of overall iterative evolution process

$$
\begin{aligned}
& \mathbf{H}_{k}=\left\{\begin{array}{llll}
h_{k}(0) & & & \\
h_{k}(1) & h_{k}(0) & & \\
h_{k}(2) & h_{k}(1) & & \\
& h_{k}(2) & \ddots & \\
& & h_{k}(0) & \\
& & h_{k}(1) & h_{k}(0) \\
& & h_{k}(2) & h_{k}(1) \\
& & & h_{k}(2)
\end{array}\right\}_{\{J+(L-1)\} \times J} \\
& \mathbf{H}_{k}^{Z P}=\left\{\begin{array}{ccc} 
& h_{k}(2) & h_{k}(1) \\
& \vdots & h_{k}(2) \\
\mathbf{H}_{k} & & \vdots \\
& h_{k}(0) & \\
& h_{k}(1) & h_{k}(0)
\end{array}\right\}_{\{J+(L-1)\} \times\{J+(L-1)\}}
\end{aligned}
$$

Note that the size of $\mathbf{H}_{k}^{Z P}$ is expanded to a square matrix with the dimension $\{J+(L-1)\} \times$ $\{J+(L-1)\}$. In this way, the channel matrix of the ZP-IDMA system is circulant. From the analysis results of [5], the ESE function can be represented as the evolution between output SNR and the users' average variance for the systems with the circulant channel matrix. This evolution is defined as SNR-variance evolution in [5]. Figure 4 shows the simplified iterative receiver. According to the analysis in [6], we give the essence of the SNR-variance techniques in ZP-IDMA system as follows.

\section{SNR-Variance Evolution Technique:}

For the ZP-IDMA system with the circulant channel matrix, we can perform iterative process of IDMA system with the average variance (denoted by $\bar{v}$ ) and SNR (denoted by $\rho$ ) for ESE and DEC. That is, the iterative process is carried out with the recursion between $\rho$ and $\bar{v}$. The transfer functions of the ESE and DEC can be denoted by $\rho=\phi(\bar{v})$ and $\bar{v}=\psi(\rho)$, respectively.

According to [5], the transfer function of ESE can be present as

$$
\rho=\phi(\bar{v})=\frac{\frac{1}{J} \sum_{j=1}^{J} \frac{\left|g_{j}\right|^{2}}{\sigma^{2}+\bar{v}\left|g_{j}\right|^{2}}}{1-\frac{\bar{v}}{J} \sum_{j=1}^{J} \frac{\left|g_{j}\right|^{2}}{\sigma^{2}+\bar{v}\left|g_{j}\right|^{2}}},
$$


where $\bar{v}$ and $g_{j}$ are

$$
\begin{aligned}
\bar{v} & =\frac{1}{J} \sum_{j=1}^{J} v_{j} \\
g_{j} & =J^{-\frac{1}{2}} \sum_{l=0}^{L-1} h_{l} \exp (-i 2 \pi j l / J) \\
i & =\sqrt{-1}
\end{aligned}
$$

We can easily get that the Eqs. (30) and (7) have the same meaning. According to [5], the value of $\rho$ can be used to predict the system performance for the iterative processing. This strategy performs well in both quasi-static fixed channels and fading channels.

In our study, we use the average variance $\bar{v}$ to replace $\rho$ as the measure of system performance prediction for simplification. Since $\bar{v}$ is in the interval $(0,1]$, the function $\rho=\phi(\bar{v})$ monotonically decreases. The details can be found in Appendix B. That means the system can obtain the best performance when the average $\bar{v}$ reaches the minimum value.

\subsection{The Relationship Between Timing Error and Average Variance}

For the system model used in this paper, we can construct the new channel coefficient $h_{K}^{\prime}$ for Eq. (21). The new channel coefficient $h_{K}^{\prime}$ is

$$
h_{K}^{\prime}=h_{K} R c_{\text {main }}\left(\Delta \tau_{K}\right) .
$$

In this approximation, the new channel matrix is also circulant as shown in (34), in which we assume there are three paths in the transmission.

$$
\mathbf{H}_{K}^{Z P}=\left\{\begin{array}{ccc} 
& h_{k}^{\prime}(2) & h_{k}^{\prime}(1) \\
\vdots & h_{k}^{\prime}(2) \\
\mathbf{H}_{K}^{\prime} & & \vdots \\
& h_{k}^{\prime}(0) & \\
& h_{k}^{\prime}(1) & h_{k}^{\prime}(0)
\end{array}\right\}_{\{J+(L-1)\} \times\{J+(L-1)\}}
$$

In (34), $\mathbf{H}_{K}^{\prime}$ can be written in a same way like (27) but using the new channel coefficients $h_{K}^{\prime}(0), h_{K}^{\prime}(1)$ and $h_{K}^{\prime}(2)$ to replace $h_{K}(0), h_{K}(1)$ and $h_{K}(2)$, respectively. Therefore, the SNR-variance evolution techniques can be adopted in our system model.

Recall the expression of ESE output for user- $K$ in (24):

$$
\operatorname{sn} r_{K}=S\left(\Delta \tau_{K}\right), \quad \Delta \tau_{K} \in\left[-\frac{T c}{2}, \frac{T c}{2}\right] .
$$

Using the results of SNR-variance in Sect. 2.2, we can establish the relationship between the timing error $\Delta \tau_{K}$ and the average variance $\overline{V_{K}}$ for the particular iterative processing results,

$$
s n r_{K}=S\left(\Delta \tau_{K}\right)=\phi\left(\overline{V_{K}}\right) .
$$

According to the analysis in Sect. 2.2, we can obtain the inverse function for the ESE function, $\rho=\phi(\bar{v})$, because of the constant monotonicity of ESE function. We define the inverse function of $\rho=\phi(\bar{v})$ as

$$
\bar{v}=\phi^{-1}(\rho)
$$




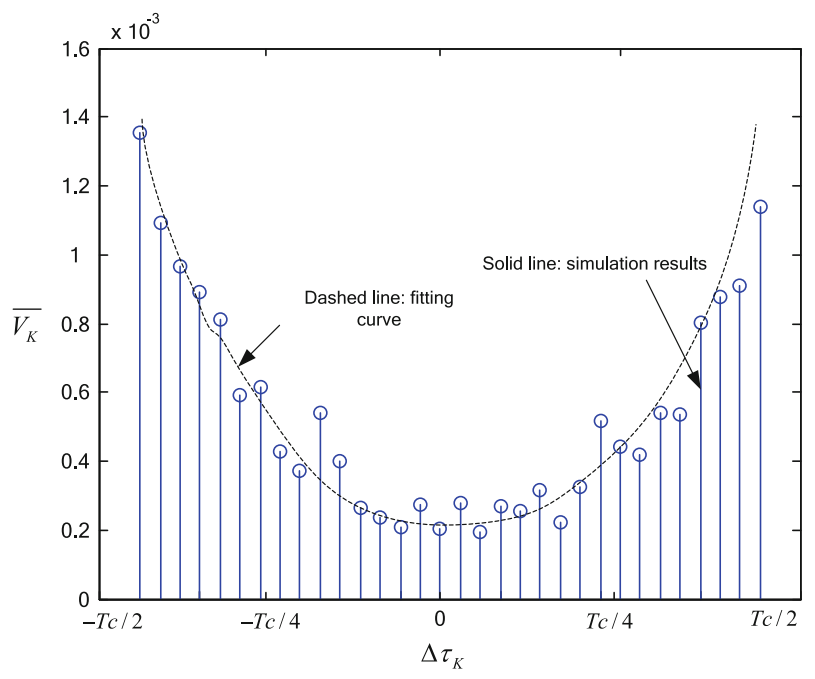

Fig. 5 The relationship between average variance and timing error

where $\phi^{-1}(\rho)$ has same monotonicity compared with its original form. Note that (36) is different from the transfer functions of DEC $\bar{v}=\psi(\rho)$. With (35) and (36), we get

$$
\overline{V_{K}}=\phi^{-1}\left(s n r_{K}\right)=\phi^{-1}\left(S\left(\Delta \tau_{K}\right)\right) .
$$

We define the function of $\overline{V_{K}}$ for $\Delta \tau_{K}$ as

$$
\overline{V_{K}}=\psi\left(\Delta \tau_{K}\right)
$$

From the characteristic of the function $S()$ and $\phi^{-1}()$ analyzed above, we can get the regularity as follows. When the timing error $\Delta \tau_{K}$ trends to zero, $\overline{V_{K}}$ gets to the minimum value, while $s n r_{K}$ gets to the maximum value. In this case, the system performance is the best. We can found that $\overline{V_{K}}$ increases with the abstract value of $\Delta \tau_{K}$ to cause the degradation of the system performance. We demonstrate this regularity with numerical results. Figure 5 shows the relationship of $\overline{V_{K}}$ and $\Delta \tau_{K}$ from our simulation results when $K=2$. The details can be found in Sect. 4.

In Fig. 5, we use dashed line to depict the fitting curve of (38), which has the profile of ' $U$ '. For the system model with an asynchronous user- $K$ who enters the system, we can predict that the timing error $\Delta \tau_{K}=0$ with high probability if we provide a scheme to let $\overline{V_{K}}$ close to the bottom of the fitting curve. This regularity provides the theoretical clues for the timing estimation. In the following, we present the details of the timing acquisition scheme.

\section{Timing Acquisition Scheme}

For the timing estimation, our target is that the $\overline{V_{K}}$ performance of the BS receiver gets to the bottom area of the fitting curve shown in Fig. 5. According to the previous analysis, the tiny timing error can cause severe performance degradation, and $\psi\left(\Delta \tau_{K}\right)$. also displays the characteristics of periodic function with the period $T=T c$. The demonstration is given below, 


$$
\begin{aligned}
\psi\left(\Delta \tau_{K}+n T c\right) & =\psi\left(\left(\Delta \tau_{K}+n T c\right) \bmod T c\right) \\
& =\psi\left(\Delta \tau_{K}\right)
\end{aligned}
$$

If we get the value of $\psi\left(\Delta \tau_{K}\right)$. for one entire period, we can easily obtain the minimum $\overline{V_{K}}$. Let the user- $K$ adjusts the transmission timing $\Delta \tau_{K}$ in a fixed step $\Delta t$ every block in the same direction, we will finally get the entire period value of $\psi\left(\Delta \tau_{K}\right)$ for $\Delta \tau_{K}$. Let $T_{b}$ denote the duration time of one block. We define the function in the time domain of the received $\overline{V_{K}}$ as:

$$
\psi_{r}(t)=\psi\left(\Delta \tau_{K}+\frac{t}{T_{b}} \Delta t_{K}\right)
$$

where $t=0, \mathrm{~T}_{b}, 2 \mathrm{~T}_{b}, \ldots$. Thus, $\psi_{r}(t)$ is the equivalent form of $\psi\left(\Delta \tau_{K}\right)$. In (40), $t=0$ means that user- $K$ initiates adding into system and starts to transmit the first data block through the uplink. $\Delta \tau_{K}+\frac{t}{T_{b}} \Delta t$ is the timing error at timing $t$. Thus, the timing error for the first block is $\Delta \tau_{K}$.

Let $T_{u}$ denote the shortest time to get the entire period of $\overline{V_{K}}$, and it can be represented as

$$
T_{u}=\frac{T c}{\Delta t} \times T_{b}
$$

Actually, we can take $T_{u}$ as the period of the received $\psi_{r}(t)$ in the BS, demonstrated as:

$$
\begin{aligned}
\psi_{r}\left(t+n T_{u}\right) & =\psi\left(\Delta \tau_{K}+\frac{t+n T_{u}}{T_{b}} \Delta t_{K}\right) \\
& =\psi\left(\Delta \tau_{K}+\frac{t}{T_{b}} \Delta t_{K}+n T c\right) \\
& =\psi\left(\Delta \tau_{K}+\frac{t}{T_{b}} \Delta t_{K}\right)=\psi_{r}(t)
\end{aligned}
$$

In order to increase the acquisition probability, we can use several periods of $T_{u}$ to achieve the timing synchronization for user- $K$. After the fixed step timing adjustment procedure, the TE can estimate where the minimum value of the received $\psi_{r}(t)$ locates. Figure 6 shows the profile of two periods $\psi_{r}(t)$ in the time domain. Let $T_{a}$ denote the duration of fixed step timing adjustment. And Fig. 6 indicates that $T_{a}=2 T_{u}$. When $t=T_{0}$, we get the minimum value of $\psi_{r}(t)$. Therefore, TE can find the best adjustment value to finish timing acquisition,

$$
\Delta \tau^{\prime}=-\frac{T_{0}}{T_{b}} \Delta t
$$

$\Delta \tau^{\prime}$ is the estimation value for $\Delta \tau_{K}$. After timing acquisition, we can perform the timing tracking with this closed-loop between BS and UE in further research. TE tracks the change of the timing error of user- $K$ with the variation of $\overline{V_{K}}$, and updates the best adjustment $\Delta \tau^{\prime}$. According to $\Delta \tau^{\prime}$, TCBG generates the timing control bits (TCB), which is sent to user- $K$ through downlink path to calibrate transmission timing.

\section{Basic TE Function:}

In our analysis, we make the approximations to get the basic profile of $\psi\left(\Delta \tau_{K}\right)$. However, in the practical systems, the received signal $\psi_{r}(t)$ gets distorted by the random noise. Therefore, we use a low pass filter to mitigate the effect of noise for the received signal $\psi_{r}(t)$. Due to the periodic characteristic for $\psi_{r}(t)$, the normalized digital pass band for the low pass filter can be computed as follows: 


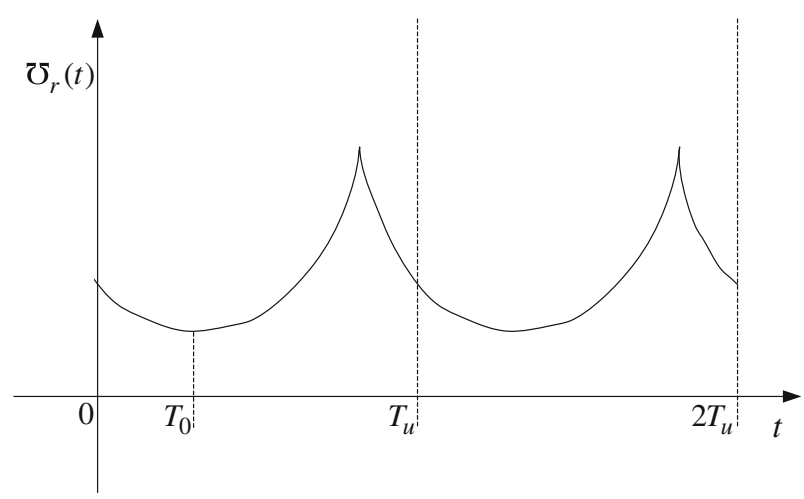

Fig. 6 Characteristics of received $r(t), T_{a}=2 T_{u} \mathrm{c}$

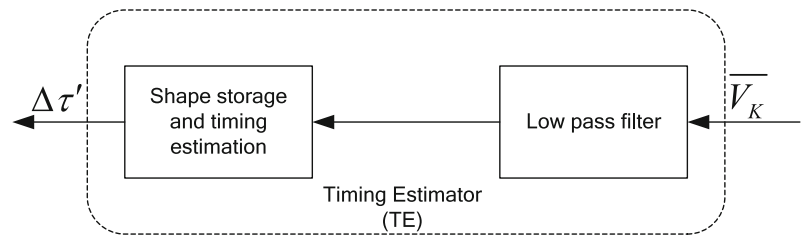

Fig. 7 Block diagram of timing estimator (TE)

$$
\omega=2 \frac{f_{u}}{f_{b}}=2 \frac{T_{b}}{T_{u}}=2 \frac{\Delta t}{T c}
$$

Figure 7 shows the operation in TE. After filtering the received signal, TE estimates the timing errors.

\section{Basic TCBG Function:}

Actually, the timing control bits generator (TCBG) is a map from timing adjustments $\Delta t$ (the fixed step) or $\Delta \tau^{\prime}$ (the estimated timing error) to the binary control messages. For the mobile station, the timing control bits of the adjustment are used to adjust its transmission timing. We assume $\Delta t$ that both known a priori at the BS and MS in the scheme. Table 1 shows the example of TCBG mapping for $\Delta t=T c / 31$.

\section{Simulation Results}

We first outline the settings and definitions used in the simulations. The quasi-static AWGN channels are used. The coefficients of multi-path channel are 1, 0.3, 0.01.

In the simulation, the $\mathrm{ZP}$ is applied in the transmission to ensure the channel matrix is circulant. The influence of ZP to the power and spectral efficiency in not considered in this paper. Each user's information symbol is encoded by a rate 1/16 repeated code. The coded signals are interleaved by randomly generated interleavers. And the roll-off coefficient $R$ of the raise cosine filter is 0.5 . Let $I T$ denote the number of iterations in the detection. The initial timing error for every user is a random value that uniformly distributes in the interval between $-T c / 2$ and $T c / 2$. For transmission, we set the length of the block $J=256$. 
Z. Wang et al.

Table 1 TCB operation map

\begin{tabular}{|c|c|c|c|c|c|}
\hline TCB & $\begin{array}{l}\text { Timing } \\
\text { adjustment }\end{array}$ & $\begin{array}{l}\text { Adjust } \\
\text { direction }\end{array}$ & TCB & $\begin{array}{l}\text { Timing } \\
\text { adjustment }\end{array}$ & $\begin{array}{l}\text { Adjust } \\
\text { direction }\end{array}$ \\
\hline 11111 & $15 T c / 31$ & Delay & 01111 & $15 T c / 31$ & Ahead \\
\hline 11110 & $14 T c / 31$ & Delay & 01110 & $14 T c / 31$ & Ahead \\
\hline 11101 & $13 T c / 31$ & Delay & 01101 & $13 T c / 31$ & Ahead \\
\hline 11100 & $12 T c / 31$ & Delay & 01100 & $12 T c / 31$ & Ahead \\
\hline 11011 & $11 T c / 31$ & Delay & 01011 & $11 T c / 31$ & Ahead \\
\hline 11010 & $10 T c / 31$ & Delay & 01010 & $10 T c / 31$ & Ahead \\
\hline 11001 & $9 T c / 31$ & Delay & 01001 & $9 T c / 31$ & Ahead \\
\hline 11000 & $8 T c / 31$ & Delay & 01000 & $8 T c / 31$ & Ahead \\
\hline 10111 & $7 T c / 31$ & Delay & 00111 & $7 T c / 31$ & Ahead \\
\hline 10110 & $6 T c / 31$ & Delay & 00110 & $6 T c / 31$ & Ahead \\
\hline 10101 & $5 T c / 31$ & Delay & 00101 & $5 T c / 31$ & Ahead \\
\hline 10100 & $4 T c / 31$ & Delay & 00100 & $4 T c / 31$ & Ahead \\
\hline 10011 & $3 T c / 31$ & Delay & 00011 & $3 T c / 31$ & Ahead \\
\hline 10010 & $2 T c / 31$ & Delay & 00010 & $2 T c / 31$ & Ahead \\
\hline 10111 & $7 T c / 31$ & Delay & 00111 & $7 T c / 31$ & Ahead \\
\hline 10110 & $6 T c / 31$ & Delay & 00110 & $6 T c / 31$ & Ahead \\
\hline 10101 & $5 T c / 31$ & Delay & 00101 & $5 T c / 31$ & Ahead \\
\hline 10100 & $4 T c / 31$ & Delay & 00100 & $4 T c / 31$ & Ahead \\
\hline 10011 & $3 T c / 31$ & Delay & 00011 & $3 T c / 31$ & Ahead \\
\hline 10010 & $2 T c / 31$ & Delay & 00010 & $2 T c / 31$ & Ahead \\
\hline 10001 & $T c / 31$ & Delay & 00001 & $T c / 31$ & Ahead \\
\hline 10000 & Zero & Hold & 00000 & Zero & Hold \\
\hline
\end{tabular}

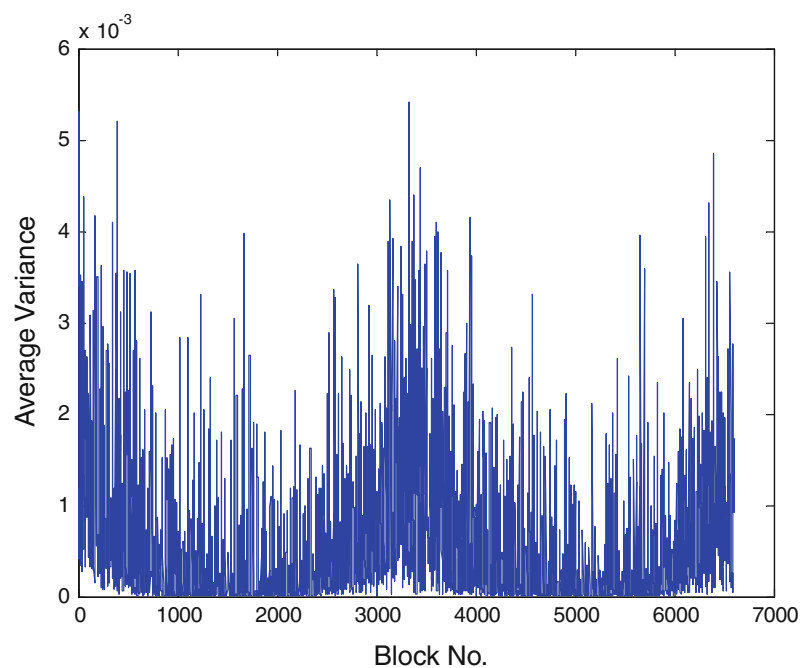

Fig. 8 The shape " $U$ " received in the BS, two cycles. $K=2, I T=15, E_{b} / N_{0}=6 d b$ 


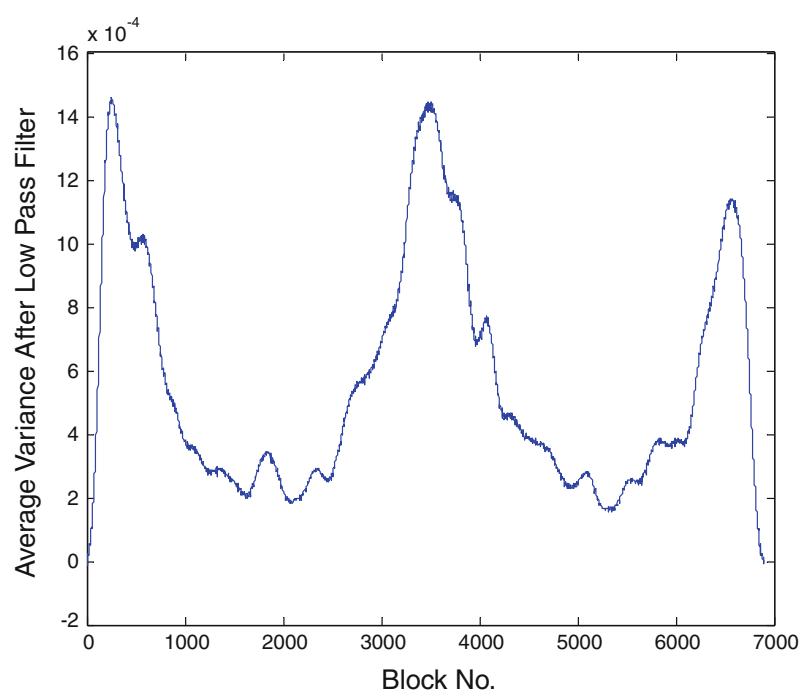

Fig. 9 The received profile "U" after low pass filter

Since the duration of timing acquisition is very short, we can assume that users are geographically fixed during the acquisition procedure. We also assume an ideal power control to obtain the equal power for every user in the BS receiver. Figure 8 shows the received signal $\overline{V_{K}}={ }_{r} \mho(t)$ in the procedure of user-2 adding into the system, which indicates $K=2$. In order to get the profile clearly, BS chooses the fixed step $\Delta t=T c / 33$ and returns the TCB to inform user- 2 to adjust its timing every 100 blocks. The initial timing error for user- 2 is $\Delta \tau_{K}=0$. Finally, we obtain the ' $\cup$ ' profile for $\overline{V_{K}}$ after timing adjustment. To make the profile smooth, we feed the output signal into the low pass filter. Figure 9 shows the results of the filtering. These two simulation results prove our approximations on signal $\psi\left(\Delta \tau_{K}\right)$ in Sect. 2.4.

In the following, we will display the performance of our proposed timing acquisition scheme. The adjustment step is $\Delta t=T c / 33$. And in the BS receiver, we configure the duration of timing adjust procedure $T_{a}=2 T_{u}$. We use $\operatorname{Dis}(K)$ which is defined in (9) to represent the distance between the true value and the estimated value. And each user adds into the system from user- 1 to user-16 one after another. Table 2 shows the timing acquisition performance for every user. From Table 2, we can see that there is no severe deterioration from the first accessed user to the last one. So the acquisition scheme is stable for different users. We also calculated the expectation and standard deviation of $\operatorname{Dis}(K)$, denoted by $E(\operatorname{Dis}(K))$ and $\operatorname{Var}(\operatorname{Dis}(k))$, respectively. The results also show the performance consistence for different users. From the previous research [4], we can see that when $|\operatorname{Dis}(K)|>T c / 4$ the system suffers severe performance loss. Therefore, we define the miss-acquisition when $|\operatorname{Dis}(K)|>T c / 4$ in the numerical simulations.

In the previous study [4], if the system can rectify the timing error into a uniform distribution that subjects to $\left(0,(T c / 16)^{2}\right)$, the performance of the system can be acceptable. Therefore, the mean and variance of $\operatorname{Dis}(K)$ can be the evaluated as the measure of acquisition performance. Table 3 shows the performance of acquisition performance with $E_{b} / N_{0}$. The miss probability and the standard deviation both decreases with $E_{b} / N_{0}$. When $E_{b} / N_{0}=0 \mathrm{~dB}$, we have the worst acquisition performance in Table 3 . With $E_{b} / N_{0}$ increas- 
Table 2 Acquisition performance for each user

\begin{tabular}{llllll}
\hline$K$ & $E_{b} / N_{0}(\mathrm{~dB})$ & Prob. in zone I & Prob. in zone II & Prob. in zone III & Miss prob. \\
\hline 1 & 6 & 0.651 & 0.216 & 0.124 & 0.021 \\
2 & 6 & 0.619 & 0.214 & 0.131 & 0.030 \\
3 & 6 & 0.702 & 0.131 & 0.095 & 0.070 \\
4 & 6 & 0.690 & 0.190 & 0.071 & 0.013 \\
5 & 6 & 0.631 & 0.261 & 0.083 & 0.024 \\
6 & 6 & 0.651 & 0.228 & 0.096 & 0.025 \\
7 & 6 & 0.663 & 0.241 & 0.084 & 0.012 \\
8 & 6 & 0.531 & 0.265 & 0.156 & 0.039 \\
9 & 6 & 0.687 & 0.216 & 0.090 & 0.012 \\
10 & 6 & 0.675 & 0.156 & 0.144 & 0.024 \\
11 & 6 & 0.711 & 0.241 & 0.036 & 0.012 \\
12 & 6 & 0.663 & 0.180 & 0.132 & 0.024 \\
13 & 6 & 0.651 & 0.216 & 0.096 & 0.036 \\
14 & 6 & 0.593 & 0.301 & 0.096 & 0.020 \\
15 & 6 & 0.631 & 0.265 & 0.045 & 0.054 \\
16 & 6 & 0.690 & 0.170 & 0.091 & 0.012 \\
\hline
\end{tabular}

Remarks:

Zone I: $-T c / 16<\operatorname{Dis}(K)<T c / 16$;

Zone II: $-T c / 8<\operatorname{Dis}(K) \leq-T c / 16$ and $T c / 16 \leq \operatorname{Dis}(K) \leq T c / 8$;

Zone III: $-T c / 4<\operatorname{Dis}(K) \leq-T c / 8$ and $T c / 8 \leq \operatorname{Dis}(K)<T c / 4$;

When $\operatorname{Dis}(K)$ locates in other area, we take it as the miss acquisition

In this situation, $E(\operatorname{Dis}(K)) \approx 0$ and $\operatorname{Var}(\operatorname{Dis}(K)) \approx\left(\frac{T c}{14}\right)^{2}$ for $K=1,2 \ldots, 16$

Table 3 Acquisition performance for different SNR

\begin{tabular}{lllll}
\hline$K$ & $E_{b} / N_{0}(\mathrm{~dB})$ & \multicolumn{1}{c}{ Mean } & Standard deviation & Miss prob. \\
\hline 8 & 0 & 0.018 & 0.197 & 0.29 \\
8 & 1 & 0.029 & 0.144 & 0.16 \\
8 & 2 & 0.022 & 0.113 & 0.13 \\
8 & 3 & -0.026 & 0.102 & 0.089 \\
8 & 4 & 0.025 & 0.094 & 0.056 \\
8 & 5 & -0.027 & 0.080 & 0.027 \\
8 & 6 & -0.026 & 0.075 & 0.028 \\
8 & 7 & -0.034 & 0.091 & 0.012 \\
8 & 8 & 0.020 & 0.066 & 0.011 \\
\hline
\end{tabular}

Remarks:

The expectations and standard deviations for $\operatorname{Dis}(K)$ are normalized by the chip duration $T c$

ing, the acquisition performance becomes better. Therefore, we can obtain that our acquisition scheme can calibrate the timing error to achieve better performance for higher SNR.

Figure 10 shows the BER performance of the asynchronous IDMA system after timing acquisition. We also set the fixed step $\Delta t=T c / 33$ and the duration time of the adjustment $T_{a}=2 T_{u}$ in this simulation. We show the system performance with different number 


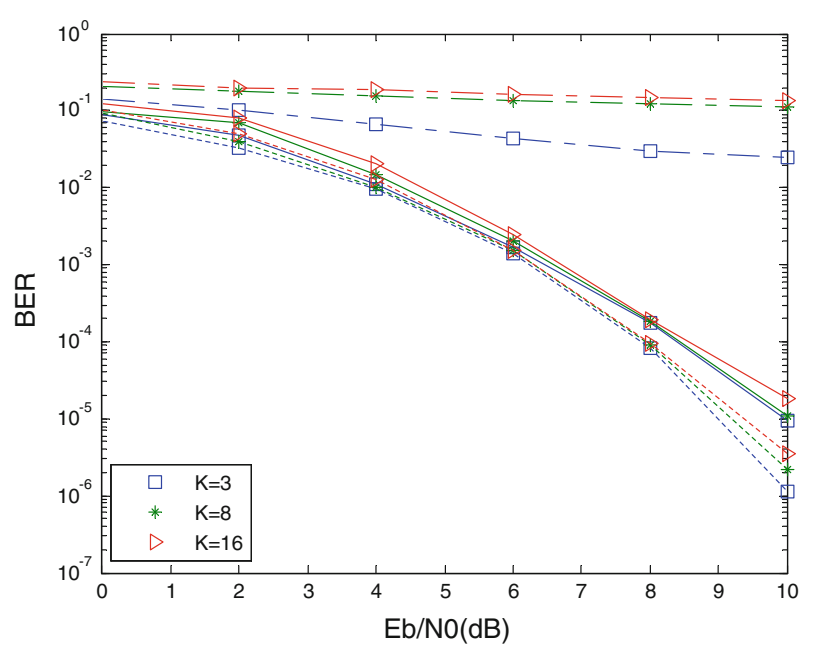

Fig. 10 Performance comparison for IDMA systems in the same quasi-static AWGN multi-path channel with different number of users. The long dashed dotted lines are for the asynchronous systems without timing calibration, and the solid lines are for the systems after timing calibration to compare with the ideal synchronous systems that are denoted by the dashed line

of users which is 3,8 and 16 , respectively. We also depict the asynchronous system performance without timing calibration to make a comparison. From this comparison, we can obtain that the system performance get enhanced after timing calibration. In Fig. 10, there is no performance floor for the proposed scheme to get the enhancement of the system performance. Furthermore, the BER performance of the perfect synchronous system is also shown in the figure. We can see that our scheme can approach the near ideal synchronous performance closely.

\section{Conclusion}

In this paper, we proposed a closed-loop timing acquisition scheme based on the SNR-variance techniques. With the help of $\mathrm{ZP}$, the average variance computed from each data block can be used in the timing error estimation. We have analyzed the relationship between timing error and the output SNR, which is demonstrated by numerical results. This analysis results can be applied in further implementations.

The simulations results show that our timing acquisition scheme performs well with acceptable acquisition probability and miss probability. And we are currently looking at the extension of this scheme to apply in the timing track for high speed users.

\section{Appendix}

Mathematical Analysis of the Monotonicity of (24)

From (23) we can obtain

$$
\begin{aligned}
s n r_{K} & =S\left(\Delta \tau_{K}\right) \\
& =\frac{h_{K}^{2} R c_{\text {main }}^{2}\left(\Delta \tau_{K}\right)}{\sum_{k=1}^{K-1}\left|h_{k}\right|^{2} \overline{V_{k}}+\left|h_{K}\right|^{2} R c_{\text {main }}\left(T c-\left|\Delta \tau_{K}\right|\right)+\sigma^{2}}
\end{aligned}
$$


We define the output of DEC as $e_{d e c}\left(x_{k}(j)\right)$. For simplicity, we use repetition code with $S$ length in our system. Ignoring the influence of the random interleaver, then $e_{d e c}\left(x_{k}(j)\right)$ can be represented as follows:

$$
\begin{aligned}
e_{d e c}\left(x_{k}(j)\right) & =\sum_{j=1}^{S} e_{e s e}\left(x_{k}(j)\right)-e_{e s e}\left(x_{k}(j)\right) \\
& =f\left(e_{\text {ese }}\left(x_{k}(j)\right)\right.
\end{aligned}
$$

In (A.1), $\overline{V_{k}}$ can be seen as a function with the variable $\Delta \tau_{K}$ as follows:

$$
\begin{aligned}
\overline{V_{k}}\left(\Delta \tau_{K}\right) & =\frac{1}{J} \sum_{j=1}^{J} \operatorname{Var}\left(x_{k}(j)\right) \\
& =\frac{1}{J} \sum_{j=1}^{J}\left[1-\tanh ^{2}\left(e_{d e c}\left(x_{k}(j)\right) / 2\right)\right] \\
& \left.=\frac{1}{J} \sum_{j=1}^{J}\left[1-\tanh ^{2}\left(e_{\operatorname{dec}}\left(x_{k}(j)\right), \Delta \tau_{K}\right) / 2\right)\right] \\
& =\frac{1}{J} \sum_{j=1}^{J}\left[1-\tanh ^{2}\left(f\left\{e_{e s e}\left(x_{k}(j)\right), \Delta \tau_{K}\right\} / 2\right)\right]
\end{aligned}
$$

where $e_{\text {ese }}\left(x_{k}(j), \Delta \tau_{K}\right)$ can be obtained by substituting (16)-(19) into (20). From (A.1) and (A.3), mathematical analysis for this function is a sophisticated issue, which is hard for us to get the close form function. Fortunately, we only care about the monotonicity of (A.1), Monte Carlo method can help us to analyze the function easily. This only takes the BS to compute $\overline{V_{k}}$ in the detection. Figure 11 shows the received $\overline{V_{k}}\left(\Delta \tau_{K}\right)$ in the entire value domain of $\Delta \tau_{K}$. The profile of this function is very similar to (36), and we can also use profile " $U$ " to approximate it.

From the simulation results, we can obtain the regularity of $\overline{V_{k}}\left(\Delta \tau_{K}\right)$ :

- From the view point of statistics theory $\overline{V_{k}}\left(\Delta \tau_{K}\right)$ is an even function,

- The profile of $\overline{V_{k}}\left(\Delta \tau_{K}\right)$ monotonically increases in the domain [0,Tc/2) for $\Delta \tau_{K}$.

Therefore, $S\left(\Delta \tau_{K}\right)$ is an even function and when $\Delta \tau_{K} \in[0, T c / 2]$ the monotonicity of (A.1) can be obtained as:

$\exists \xi \rightarrow 0^{+}$to make

$$
\begin{aligned}
& S\left(\Delta \tau_{K}+\xi\right)-S\left(\Delta \tau_{K}\right) \\
& =\frac{h_{K}^{2} R c_{\text {main }}^{2}\left(\Delta \tau_{K}+\xi\right)}{\sum_{k=1}^{K-1}\left|h_{k}\right|^{2} \overline{V_{k}}}\left(\Delta \tau_{K}+\xi\right)+\left|h_{K}\right|^{2} R c_{\text {main }}\left(T c-\Delta \tau_{K}-\xi\right)+\sigma^{2} \\
& \quad-\frac{h_{K}^{2} R c_{\text {main }}^{2}\left(\Delta \tau_{K}\right)}{\sum_{k=1}^{K-1}\left|h_{k}\right|^{2} \overline{V_{k}}}\left(\Delta \tau_{K}\right)+\left|h_{K}\right|^{2} R c_{\text {main }}\left(T c-\Delta \tau_{K}\right)+\sigma^{2}
\end{aligned}
$$

Let

$$
A_{1}=\sum_{k=1}^{K-1}\left|h_{k}\right|^{2} \overline{V_{k}}\left(\Delta \tau_{K}+\xi\right)+\left|h_{K}\right|^{2} R c_{\text {main }}\left(T c-\Delta \tau_{K}-\xi\right)+\sigma^{2}
$$




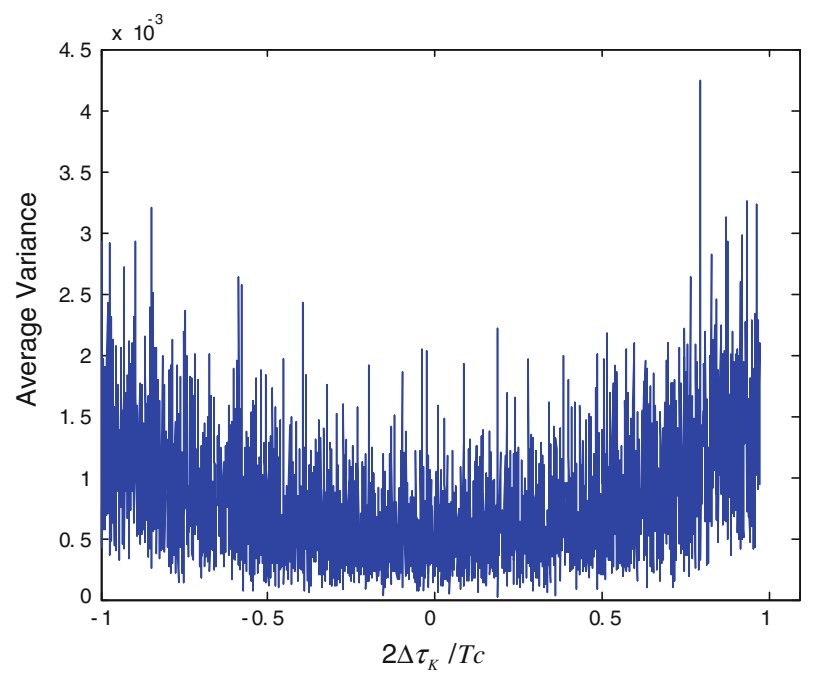

Fig. 11 The average variance for the accessed users when the timing error of user- $K$ varies from $-T c / 2$ to $T c / 2$

and

$$
A_{2}=\sum_{k=1}^{K-1}\left|h_{k}\right|^{2} \overline{V_{k}}\left(\Delta \tau_{K}\right)+\left|h_{K}\right|^{2} R c_{\text {main }}\left(T c-\Delta \tau_{K}\right)+\sigma^{2},
$$

and we can easily get that: $A_{1}>0$ and $A_{2}>0$, and also $A_{1}>A_{2}$. Therefore, (A.4) can be rewritten as

$$
\begin{aligned}
S & \left(\Delta \tau_{K}+\xi\right)-S\left(\Delta \tau_{K}\right) \\
& =h_{K}^{2}\left(\frac{R c_{\text {main }}^{2}\left(\Delta \tau_{K}+\xi\right)}{A_{1}}-\frac{R c_{\text {main }}^{2}\left(\Delta \tau_{K}\right)}{A_{2}}\right) \\
& =h_{K}^{2} \frac{R c_{\text {main }}^{2}\left(\Delta \tau_{K}+\xi\right) A_{2}-R c_{\text {main }}^{2}\left(\Delta \tau_{K}\right) A_{1}}{A_{1} A_{2}} \\
& <0 .
\end{aligned}
$$

From the analysis above, when $\Delta \tau_{K} \in[0, T c / 2], S\left(\Delta \tau_{K}\right)$ monotonically decreases. And when $\Delta \tau_{K} \in[-T c / 2,0], S\left(\Delta \tau_{K}\right)$ monotonically increases. $S\left(\Delta \tau_{K}\right)$ gets its maximum value at $\Delta \tau_{K}=0$.

Mathematical Analysis of the Monotonicity of (29)

From (29), we define

$$
\mu(\bar{v})=\frac{1}{J} \sum_{j=1}^{J} \frac{\left|g_{j}\right|^{2}}{\sigma^{2}+\bar{v}\left|g_{j}\right|^{2}}
$$

Substituting (B.1) into (29), and then we get

$$
\rho=\phi(\bar{v})=\frac{\mu(\bar{v})}{1-\mu(\bar{v}) \bar{v}}
$$


To get the monotonicity of (29), we differentiate (B.2) as follows:

$$
\begin{aligned}
\frac{d(\phi(\bar{v}))}{d \bar{v}} & =\frac{d\left(\frac{\mu(\bar{v})}{1-\mu(\bar{v}) \bar{v}}\right)}{d(\mu(\bar{v}))} \times \frac{d(\mu(\bar{v}))}{d \bar{v}} \\
& =\frac{1-\mu(\bar{v}) \bar{v}+\mu(\bar{v})^{2}}{(1-\mu(\bar{v}) \bar{v})^{2}} \times \frac{1}{J} \sum_{j=1}^{J} \frac{-\left|g_{i}\right|^{2}}{\left(\sigma^{2}+\bar{v}\left|g_{i}\right|^{2}\right)^{2}}
\end{aligned}
$$

where

$$
\begin{aligned}
\mu(\bar{v}) \bar{v} & =\frac{1}{J} \sum_{j=1}^{J} \frac{\left|g_{i}\right|^{2} \bar{v}}{\sigma^{2}+\left|g_{i}\right|^{2} \bar{v}}=\frac{1}{J} \sum_{j=1}^{J} \frac{1}{\frac{\sigma^{2}}{\left|g_{i}\right|^{2} \bar{v}}+1} \\
& <\frac{1}{J} \sum_{j=1}^{J} \frac{1}{1}=1
\end{aligned}
$$

Therefore, we can obtain that

$$
\frac{d(\phi(\bar{v}))}{d \bar{v}}<0
$$

The function $\phi(\bar{v})$ monotonically decreases in the value domain of $\bar{v}, 1 \geq \bar{v}>0$.

Acknowledgments This work was fully supported by the Nature Science Founding of China [Project No. 60872030, 61101033 and 61070696]; China Postdoctoral Science Foundation funded project [Project No. 20100471672], and Sichuan Province Science Foundation for Youths [No. 2011JQ0034].

\section{References}

1. Ping, L., Liu, L., Wu, K., \& Leung, W. K. (2006). Interleave-division multiple-access. IEEE Transactions on Wireless Communication, 5(4), 938-947.

2. Ping, L., Liu, L., Wu, K., \& Leung, W. K. (2003). Interleave-division multiple-access (IDMA) communications. In Proceedings of 3rd international symposium on turbo codes and related topics (pp. 173-180).

3. Liu, L., Leung, W. K., \& Ping, L. (2003). Simple chip-by-chip multi-user detection for CDMA systems. In Proceedings of IEEE VTC'2003-Spring, Jeju, Korea (pp. 2157-2161)

4. Wang, Z., Hu, J., \& Xiong, X. (2009). Effect of sample-timing error on performance of interleavedivision multiple access system. In 7th International conference on information and communication systems (pp. 1-6), Macau.

5. Yuan, X., Guo, Q., Wang, X., \& Ping, L. (2008). Evolution analysis of low-cost iterative equalization in coded linear systems with cyclic prefixes. IEEE Journal on Selected Areas in Communications, 26(2), 301-310.

6. Guo, Q., Yuan, X., \& Ping, L. (2008). Single- and multi-carrier IDMA schemes with cyclic prefixing and zero padding techniques. European Transactions on Telecommunications, 19(5), 1-11.

7. Yuan, X., Li, H., Ping, L., \& Lin, X. (2008). Precoder design for ISI channels based on iterative LMMSE equalization. In 5th International Symposium on Turbo Codes and Related Topics (pp. 198-203).

8. Morelli, M., Kuo, C. C. J., \& Pun, M. O. (2007). Synchronization techniques for orthogonal frequency division multiple access (OFDMA): A tutorial review. Proceedings of the IEEE, 95(7), 1394-1427.

9. Muquet, B., De Courville, M., Duhamel, P., Giannakis, G. B., \& Magniez, P. (2002). Turbo demodulation of zero-padded OFDM transmissions. IEEE Transactions on Communications, 11, 1725-1728.

10. Wang, Z., Ma, X., \& Giannakis, G. B. (2004). OFDM or single-carrier block transmissions?. IEEE Transactions on Communications, 3, 380-394.

11. Muquet, B., Wang, Z., Giannakis, G. B., De Courville, M., \& Duhamel, P. (2002). Cyclic prefixing or zero padding for wireless multi-carrier transmissions?. IEEE Transactions on Communications, 12, 21362148 . 
12. Boutros, J., \& Caire, G. (2002). Iterative multi-user joint decoding: Unified framework and asymptotic analysis. IEEE Transactions on Information Theory, 48, 1772-1793.

13. Caire, G., Muller, R. R., \& Tanaka, T. (2004). Iterative multiuser joint decoding: Optimal power allocation and low-complexity implementation. IEEE Transactions on Information Theory, 50, 1950-1973.

\section{Author Biographies}

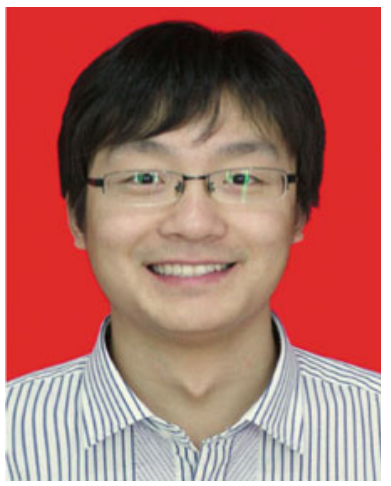

Zhao Wang received the BS degree and ME degree from the University of Electronic Science and Technology of China (UESTC), Chengdu, in 2007 and 2010, respectively. He is currently pursuing the $\mathrm{PhD}$ degree in Royal Institute of Technology (KTH).

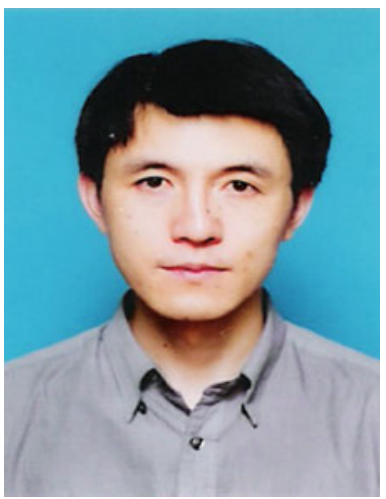

JianHao Hu received the $\mathrm{PhD}$ degree in communications from UESTC, Chengdu, China, in 1999. From 1999 to 2000, he was a postdoctoral with City University of Hong Kong, and researched on mobile communication systems. From 2000 to 2004, he joined the University of Hong Kong, Hong Kong, where he worked on CDMA2000 system development in the university's $3 \mathrm{G}$ research center. Since 2005, he joined the National Key Lab. of Communications of UESTC, as a professor. His research interests include wireless communication and VLSI.

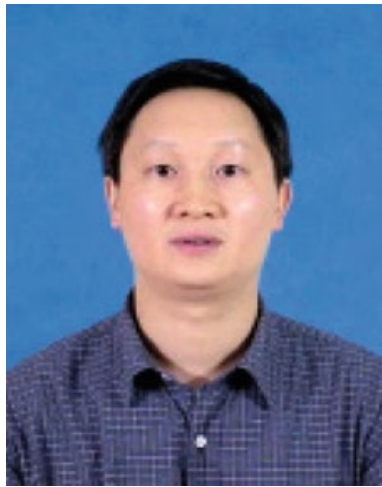

XingZhong Xiong received the BE degree in electronic engineering from the Sichuan University of Science \& Engineering (SUSE), Sichuan, China, in 1996, the ME degree in signal and information processing from the University of Electronic Science and Technology of China (UESTC), Chengdu, China, in 2006, and the PhD degrees in communication and information system from UESTC, in 2009. He is currently a professor in the SUSE. His research interests include signal processing and multiple access techniques in communication systems. 


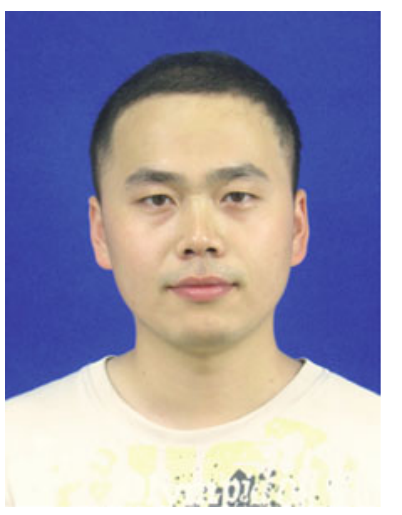

Jie Song received the BS degree and ME degree from the University of Electronic Science and Technology of China (UESTC), Chengdu, in 2007 and 2010, respectively. He is currently a system engineer in Huawei, Chengdu, China. 